\title{
CASE REPOR'T
}

\section{Blistering rash in a young male child}

\author{
C Sanders, MB ChB; H Potgieter, MD, FCPaed \\ Paediatrics Department, Leratong Hospital, Johannesburg, South Africa
}

Corresponding author: C Sanders (cathrynsand@gmail.com)

A 3-year-old child was brought to the casualty department at Leratong Hospital, Johannesburg, South Africa, with severe bullous eruptions on the distal portions of both the upper and lower limbs. He had visited three different general practitioners prior to presentation and had been given several drugs and ointments, including three different classes of antibiotics. During his admission, the diagnosis of linear IgA bullous dermatitis was made. Dapsone and prednisone were given and he recovered well.

S Afr Med J 2017;107(7):578-580. DOI:10.7196/SAMJ.2017.v107i7.12407

Linear IgA bullous dermatosis (LABD) is an autoimmune skin disorder in which blisters form as a result of a split between the epidermis and dermis. The first cases of LABD date from 1901 - 1905, when it was still considered to be part of the spectrum of dermatitis herpetiformis. Only 80 years later was it recognised as a distinct clinical entity. ${ }^{[1]}$

IgA autoantibodies react to components of the hemidesmosomes and basement membrane. ${ }^{[2]}$ The blisters are classically tense, indicating that they are of subepidermal origin as opposed to the flaccid bullous lesions of epidermal origin. ${ }^{[3]}$

The name LABD arises from the finding of a line of IgA antibodies below the epidermis on immunofluorescence examination of a skin biopsy specimen. ${ }^{[3]}$

\section{Case report}

A 3-year-old boy was brought to the emergency department at Leratong Hospital, Johannesburg, South Africa, with a 1-week history of progressively worsening blisters on his arms and legs. He was unexposed to HIV and had previously been well, with no prior hospital admission.

Two weeks before the appearance of the rash, the patient had suffered from a fever and vomiting. His mother took him to a private general practitioner (GP), who prescribed cyclizine (an antiemetic), Goldgesic syrup (mainly paracetamol), amoxicillin plus clavulanic acid, and a co-trimoxazole suspension.

After the patient had been on the above treatment for about 3 days, his mother noticed a rash comprising small blisters on his arms and legs. A different GP was consulted, who prescribed griseofulvin, retapamulin ointment, Tetmosol soap, cloxacillin and chlorpheniramine maleate, with no advice to stop the previously prescribed drugs.

The rash got progressively worse, so when the mother took the child to a third GP, she was advised to take him to hospital.

On presentation to Leratong Hospital the patient was well, with vital signs within normal limits and no remarkable findings on systemic examination.

On examination of the skin, tense, deep bullae with surrounding vesicles on an erythematous base were present on the distal extremities. There were areas of central necrosis and a tendency to coalesce (Fig. 1). The mucous membranes were not involved and the palms and soles were spared. Around the left wrist and right lower limb, the lesions had formed a 'string of pearls' sign (Fig. 1). There

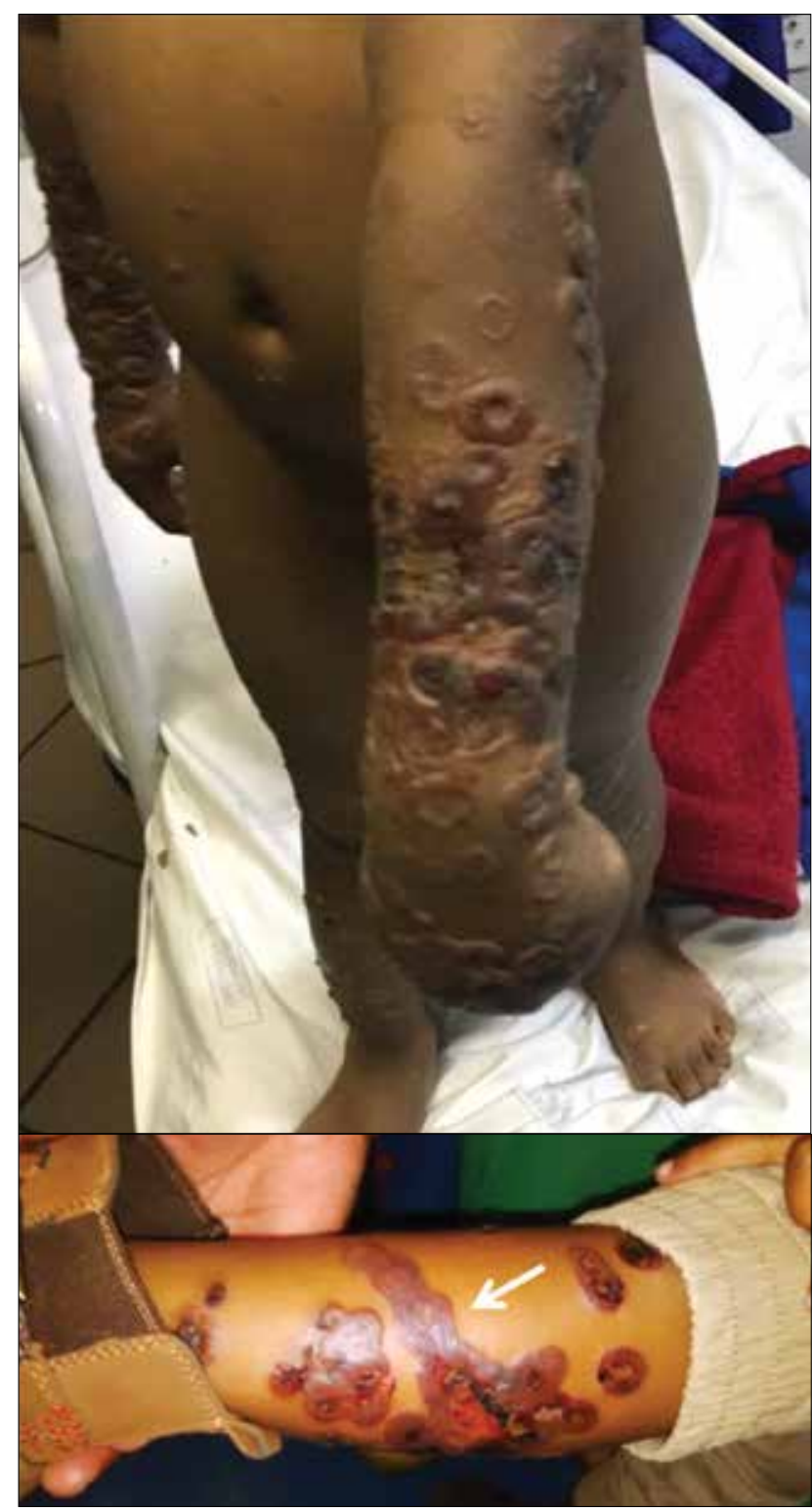

Fig. 1. The lesions on presentation, including the 'string of pearls' sign (white arrow). 


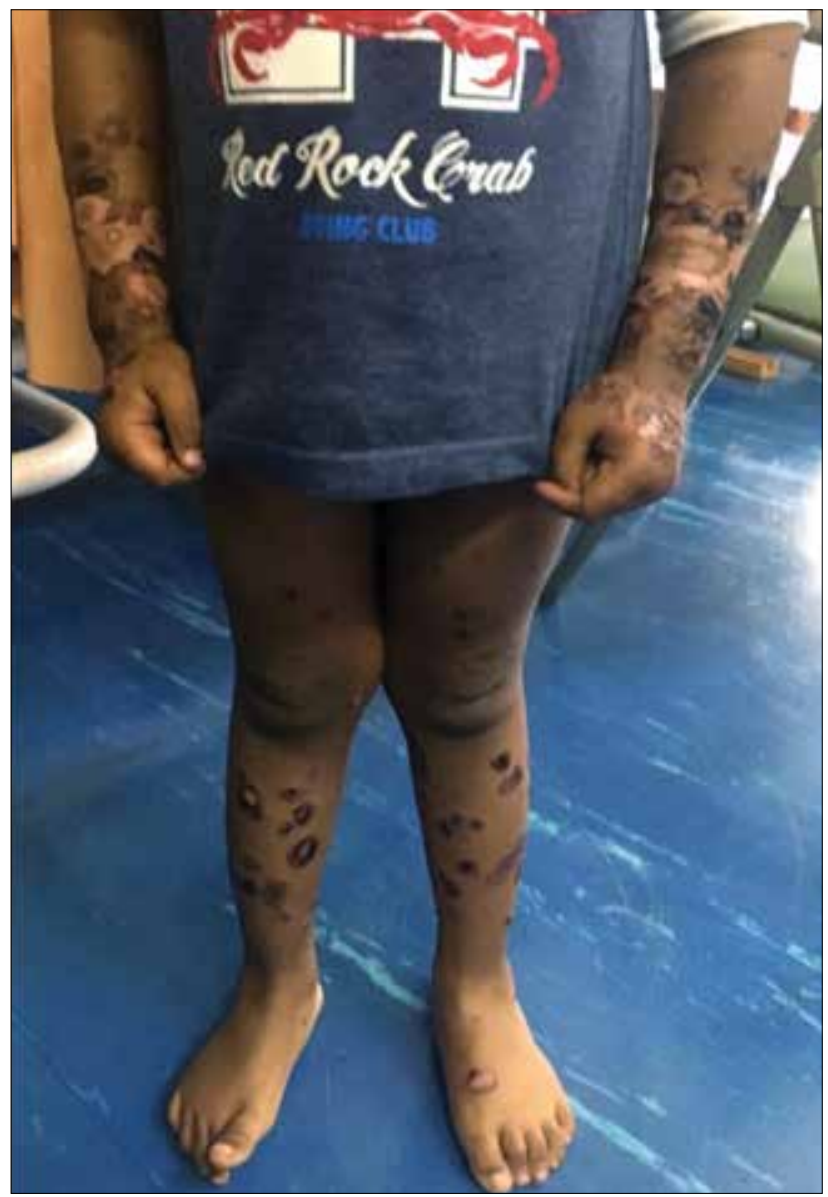

Fig. 2. Clinical improvement after 1 week's treatment with prednisone and dapsone.

were a few smaller vesicular lesions on the lower abdomen and around the anus. The patient complained that the lesions were itchy, and the lesions around his anus were painful, making it difficult for him to pass a stool.

The patient was admitted and assessed as suffering from a possible drug reaction. All medication was stopped and he was referred to the Dermatology Department at Baragwanath Hospital for consultation and a biopsy.

The dermatologist's differential diagnosis was LABD, followed by bullous erythema multiforme. The treatment given was a combination of prednisone and dapsone, as well as topical steroids and emollients to use on the affected areas. The patient responded well to treatment, with the lesions showing vast improvement after 1 week of the prescribed medication (Fig. 2).

The patient was followed up at the Leratong outpatient department, and 6 months after the initial presentation the lesions had almost completely healed, with evidence of post-inflammatory hyperpigmentation (Fig. 3). Review of the histological findings and direct immunofluorescence examination confirmed the diagnosis of linear IgA bullous dermatosis, an intact skin punch biopsy showing $1-2+$ linear staining in the region of the epidermal basement membrane.

\section{Discussion}

Linear IgA dermatosis is a rare autoimmune bullous disease, yet the most common autoimmune bullous disease in children. ${ }^{[1]}$ The aetiological factor triggering the autoimmune mechanism of the disease is unknown. ${ }^{[1]}$

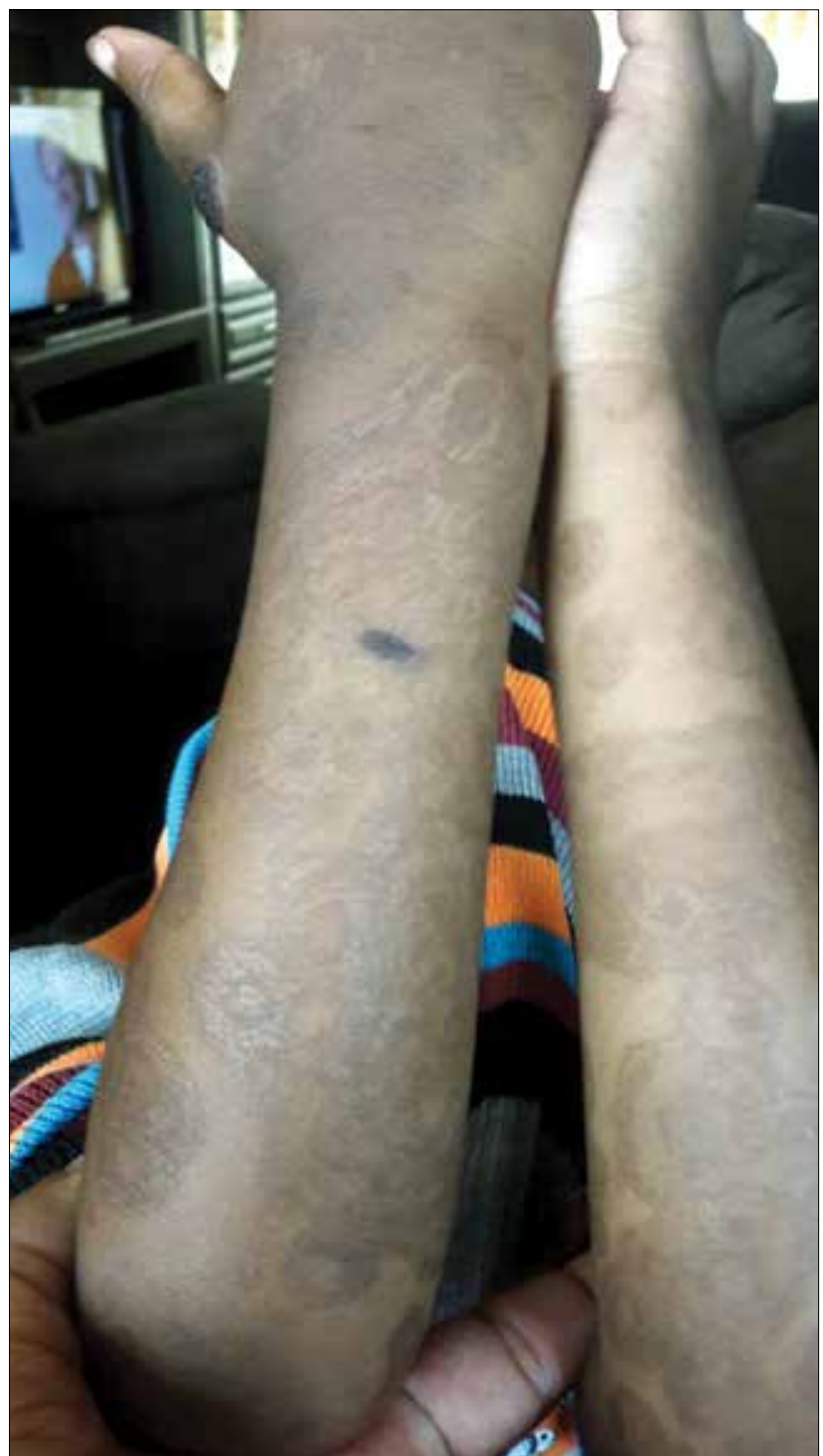

Fig. 3. Almost complete healing 6 months after initial presentation.

The typical clinical presentation of LABD is of small vesicles or bullae, which are annular and may develop into a target shape. There is a tendency for new vesicles to form in a ring around the old lesion, giving the 'string of beads/pearls' sign, which was present in our patient.

It may be difficult to distinguish LABD clinically from dermatitis herpetiformis. They are both blistering diseases and on immunofluorescence microscopy show evidence of IgA deposition; however, dermatitis herpetiformis is associated with a gluten-sensitive enteropathy (coeliac disease). ${ }^{[4]}$

The presentation of LABD can follow a viral illness or exposure to a drug. The most frequently associated drugs include vancomycin, diclofenac and co-trimoxazole, but the most consistent association described in the literature is with vancomycin. ${ }^{[1,3,5]}$

The diagnosis is made on examination of a skin biopsy specimen, where an infiltration of neutrophils and eosinophils is seen under the epidermis. Direct immunofluorescence examination reveals IgA along the basement membrane in a linear pattern.

Most cases reported in the literature had a similar outcome to that in our patient, complete remission being achieved following a course of prednisone and dapsone. ${ }^{[1]}$ One such case report described a 10-month-old white child with LABD with a similar presentation to 
our patient, but with no fever or mucosal signs. However, in contrast to our case, there was no causal relationship with drug exposure or viral illness to explain the occurrence of the lesions. ${ }^{[1]}$

In a similar case report, where an infant developed LABD but failed to respond to prednisone, mycophenolic acid was used with a good clinical outcome. ${ }^{[6]}$ This drug should be used with caution, however, as it can have adverse effects such as myelosuppression, infections and severe diarrhoea.

When dapsone is contraindicated, e.g. in patients with glucose-6phosphatase dehydrogenase deficiency, haemolytic anaemia or severe bone marrow suppression, LABD has been successfully treated with flucloxacillin with a decreased incidence of remission, provided the treatment was started early on in the course of the disease..$^{[7]}$

\section{Conclusion}

This case illustrates the importance of knowing about LABD, the differential diagnosis of which includes bullous disease of childhood and erythema multiforme. It also highlights the danger of prescribing a 'shopping list' of drugs/antibiotics, which makes it difficult to identify the offending drug if an adverse drug reaction does develop. It also emphasises the importance of timeous referral to appropriate specialist support for early recognition, relevant special investigations and treatment of unusual clinical presentations.

Acknowledgements. None.

Author contributions. Written by CS, with contributions from HP.

Funding. None.

Conflicts of interest. None.

1. De Souza BC, Tebcherani A. Linear IgA bullous dermatosis: Report of an exuberant case. An Bras Dermatol 2013;88(6 Suppl 1):S67-S70. http://doi.org/10.1590/abd1806-4841.20132154

2. Burge S. Oxford Handbook of Medical Dermatology. 1st ed. London: Oxford, 2011.

DermNet New Zealand. Linear IgA bullous disease. 2015. www.dermnetnz.org/topics/linear-igabullous-disease (accessed 11 November 2016).

4. Kirtschig G, Wojnarowska F. Autoimmune blistering diseases: An up-date of diagnostic methods and investigations. Clin Exp Dermatol 1994;19(2):97-112. http://doi.org/10.3238/arztebl.2011.0405

5. Nousari HC, Kimyai-Asadi, A, Caeiro JP, et al. Clinical, demographic and immunohistological features of vancomycin induced linear IgA bullous disease of the skin: Report of two cases and review of the literature. Medicine (Baltimore) 1999;78(1):1-8.

6. Farley-Li J, Mancini A. Treatment of linear IgA bullous dermatosis of childhood with mycophenolate mofetil. Arch Dermatol 2003;139(9):1121-1124. http://doi.org/10.1001/archderm.139.9.1121

7. Alajlan A, Al-Khawajah M, Al-Sheikh O, et al. Treatment of linear IgA bullous dermatosis of childhood with flucloxacillin. J Am Acad Dermatol 2006;54(4):652-656. http://doi.org/10.1016/j. jaad.2005.11.1102

Accepted 14 March 2017 